IAU Colloquium 190 on Magnetic Cataclysmic Variables

ASP Conference Series, Vol. 315, 2004

Sonja Vrielmann \& Mark Cropper, eds.

\title{
The Characteristics of Magnetic CVs in the Period Gap
}

\author{
Gaghik Tovmassian, Sergey Zharikov \\ Observatorio Astronómico Nacional, Instituto de Astronomía, UNAM, \\ P.O. Box 439027, San Diego, CA, USA 92143-9027
}

Ronald Mennickent

Dpto. de Fisica, Fac. de Cs. Fisicas y Mat., Universidad de

Concepcion, Casilla 160-C, Concepcion, Chile

Jochen Greiner

Max-Planck-Institute for Extraterrestrial Physics (MPE), 85741

Garching, Germany

\begin{abstract}
We have observed several magnetic cataclysmic variables located in the range between 2 and 3 hours, known as the period gap. This work was prompted by the recent discovery of RX J1554.2+2721. It has 2.54 hours orbital period and shows almost pure cyclotron continuum in a low luminosity state, similar to HS1023+3900, HS0922+1333 and RBS206. These are low accretion rate polars (LARPs) known to have mass transfer rates of order of a few $10^{-13} \mathrm{M}_{\odot} /$ year. The aim of the study was to find out, if magnetic systems filling the period gap are in any way different from their counterparts outside that range of periods. The only significant difference we encounter is a much higher number of asynchronous magnetic systems towards longer periods than below the gap.
\end{abstract}

\section{Introduction}

Cataclysmic Variables (CVs) are close interactive binaries with the majority of their orbital periods distributed between 80 minutes and 10 hours. The bulk of CVs (Dwarf Novae and some non-magnetic Nova-likes) show a bimodal distribution of orbital periods with a well pronounced deficiency of systems between 2 and 3 hours, known as the period gap. For a long time it's been argued and now commonly accepted that the magnetic systems does not follow that pattern and the distribution of magnetic CVs (MCVs) does not show such bi-modality (Webbink \& Wickramasinghe 2002). The explanation that they suggest is based on magnetic breaking models that allow narrowing of the period gap for sufficiently high magnetic moment of the primary star, immediately after which the mass transfer is driven by gravitational waves. The model provides a good description of the orbital period distribution of MCVs, but it predicts much higher rates of mass transfer than has been observed in a few MCVs in the period gap and around it. 
Tovmassian et al.

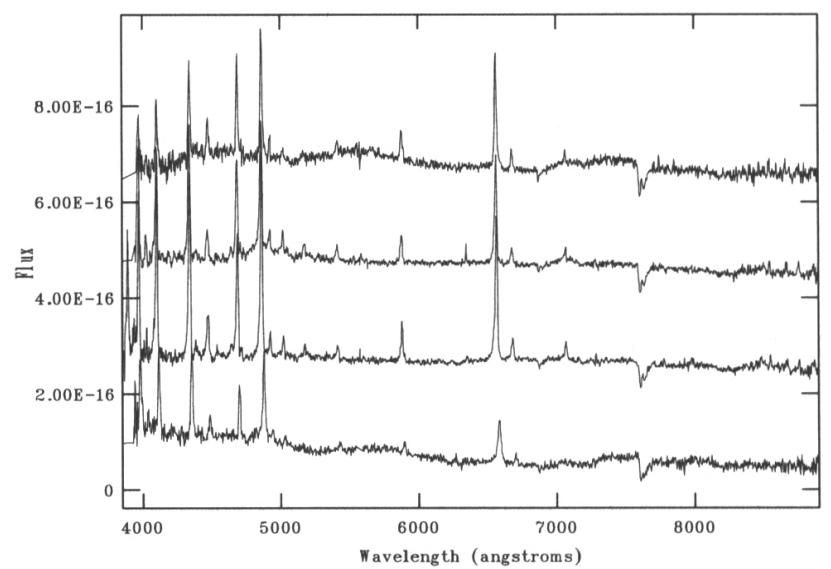

Figure 1. Spectra of V381 Vel in different phases

We were able to conduct spectrophotometry of a sample of MCVs in the period gap to compare their characteristics with others. We also observed one object that lies above the period gap but also shows an extremely low mass transfer rate.

The question is whether the mass transfer decrease is directly associated with the period gap, and thus detection of MCVs in the gap is a matter of selection effects (primarily X-ray emission), or if it is an intrinsic characteristic of particular systems.

\section{Observations and basic characteristics of the selected objects}

There are 51 systems with periods between 2 and 3 hours in the living edition of the Downes (1997/2002) catalog of Cataclysmic Variables and 25 of them are proven to be magnetic systems. We collected low resolution spectrophotometric data on 3 of these. Although they constitute only a small fraction of the systems inside the period gap, they exhibit wide range of features characteristic of Polars. We also observed HS0922+1333 which is remarkable for its low mass accretion rate, its pure cyclotron spectrum and its period longward of the gap.

The observations were conducted mainly at the $2.1 \mathrm{~m}$ telescope of OAN in Mexico. One object (V381Vel) was observed from La Silla with 3.5m NTT (for details of observations see Tovmassian et al. (2003a, 2003b).

\section{1. $\quad$ V381 Vel $=$ RX J1016.9-4103}

The optical counterpart of this Polar was identified by Greiner \& Schwarz (1998). They estimated $P_{\text {orb }}=134$ minutes. The system has one pole with a magnetic field of $B=52 \mathrm{MG}$ or $41 \mathrm{MG}$. Vennes et al. (1999) later cited a shorter $\mathrm{P}_{\text {orb }}=122$ min, without elaborating on the other properties. We observed one full period in April 1999 without being aware of the discrepancy. Complete orbital coverage allowed us to conclude that the system actually has two active poles. At orbital phases $0.20-0.25$ we can see mix of both poles, as Greiner \& Schwarz (1998) did. At phase 0.5 no cyclotron humps are apparent. Later at $\phi=0.7$ the $2 \mathrm{nd}$ 

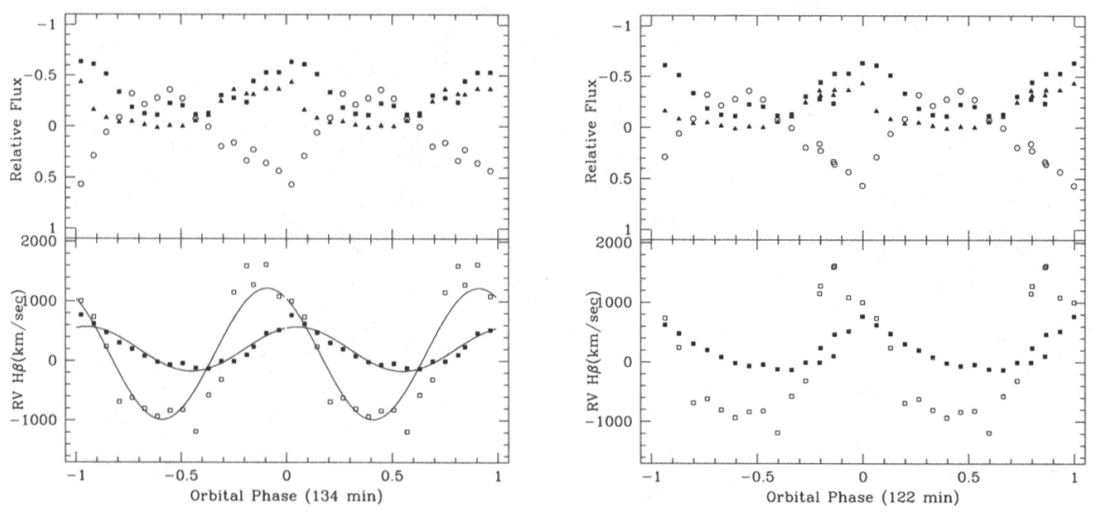

Figure 2. Radial velocities of $\mathrm{H} \alpha$ line and fluxes of cyclotron lines and continuum of V381 Vel folded with orbital (left) and spin (right) periods.
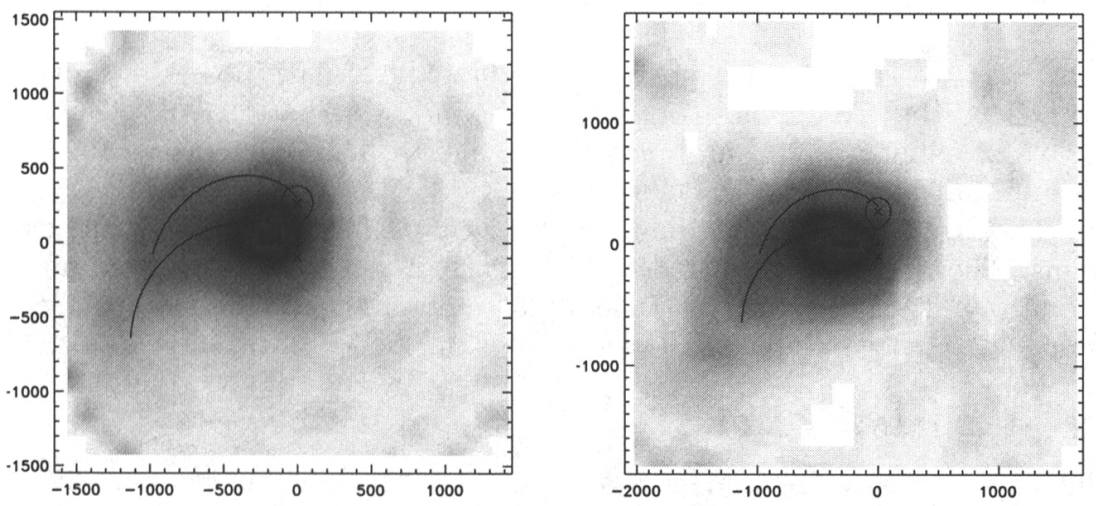

Figure 3. Doppler maps of V381 Vel. H $\alpha$ in the left panel and He II in the right.

harmonic of the $41 \mathrm{MG}$ pole dominates. Finally at phase 0.95 through 0.1 the stronger $52 \mathrm{MG}$ pole is seen (see Figure 1).

The emission lines were successfully separated into two components. None of these components arises from the irradiated secondary star. Rather they are emitted from different parts of the accretion stream. Folding the data with both announced periods and fitting a sine curve strongly favors 134 minutes as the orbital period of the system (Figure 2, lower left panel). Next, we integrated the flux in three different bands. They were selected to reflect the flux variation in the continuum relatively free of emission lines (including cyclotron lines), and two other narrow bands centered on the cyclotron features. The folding of the continuum fluxes, presumably from the self-eclipsing WD, with the orbital (134 minutes) period is poor (Figure 2, upper left panel). The same data folded with 122 minutes period greatly improves the light curve, but distorts the radial 

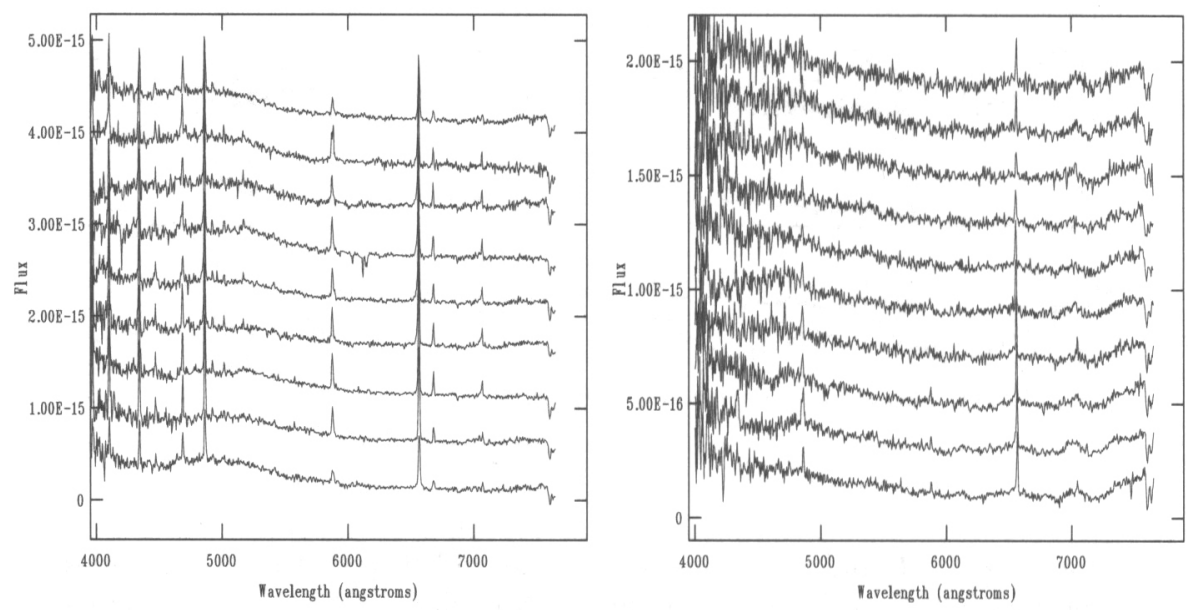

Figure 4. Spectra of RXJ1554.2+2721 in high (left) and low (right) states around the orbital period.

velocity curve (right panel, note the overlapping of data points at $\phi 0.8-0.9$ ). We believe that this Polar is not synchronized and that $P_{\text {orb }}=134$ minutes, while 122 minutes is the spin period of the WD. The difference is about $10 \%$, which in unprecedented for Polars.

The orbital phasing can be independently confirmed by the Doppler tomograms. The characteristic pattern of the mass transfer flow is oriented precisely, as one would expect with the right parameters and phasing (Figure 3 ). Once again the absence of an irradiated secondary in the V381 Vel should be stressed.

\subsection{RX J1554.2+2721}

This object was recently identified as a Polar (Tovmassian et al. 2001). We estimated $\mathrm{P}_{\text {orb }}=2.753$ hours. Shortly after, Thorstensen \& Fenton (2002) refined it to 2.53 hours, placing it exactly in the center of the period gap. Its main features include frequent switches from the high to the low luminosity states, extremely low mass transfer rate in the low state and a striking similarity to other LARPs (HS0922+1333, HS1023+3900 and RBS 206). This latter conclusion was based upon a single spectrum obtained in the low luminosity state. We re-observed RXJ1554.2+2721 until we found it in a low state again. These new observations confirmed that the object goes from the high state to the low within months. We obtained full orbital coverage of the system in the high and in the low state. We confirmed the estimate of the magnetic field strength of order of $B=30 \mathrm{MG}$. The object probably has a weaker second actively accreting pole. In the Figure 4 we present samples of spectra of RX J1554.2+2721 at different phases in the low and high states.

Here again we separated the emission lines into two components. In this case however, the dominant component arises from the irradiated secondary star, an M4 red dwarf. This component is basically the only line emission observed in the low state. The stream is not detected or is extremely faint. Doppler 


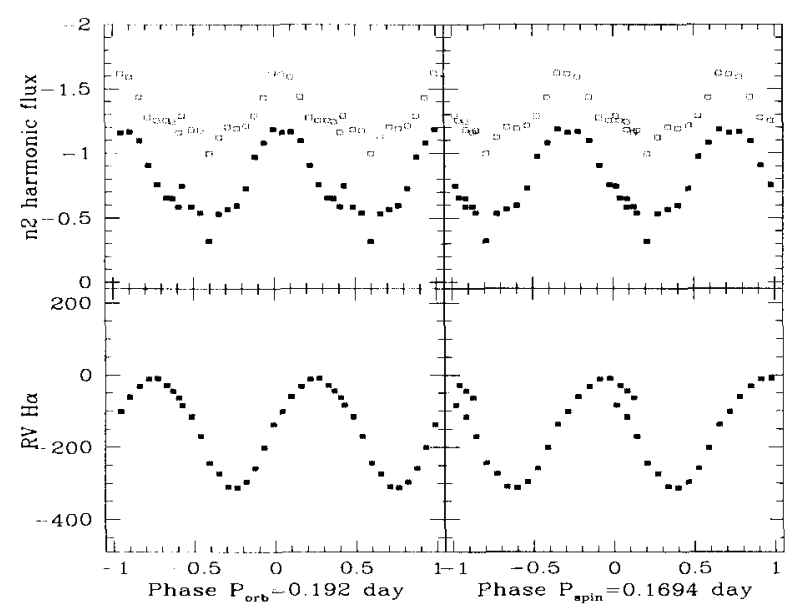

Figure 5. Radial velocities of $\mathrm{H} \alpha$ line and fluxes of cyclotron lines of HS0922+1333 folded with orbital (left) and spin (right) periods.

maps in Tovmassian et al. (2001) and Thorstensen \& Fenton (2002) testify to the presence of a strongly irradiated secondary and a weak stream contribution unlike in V381 Vel. The system seems to be synchronized: at least no deviations are detected at our precision level. These are preliminary results and we expect to obtain more useful information from the wealth of data that was acquired.

\subsection{HS1023+3900}

We observed HS1023+3900 along with RXJ 1554.2+2721 in January \& April 2002 primarily to check if it undergoes states of higher luminosity. In both occasions the object looked exactly as described in Reimers, Hagen \& Hopp (1999). There is a continuing evidence of an irradiated secondary ( $\mathrm{H} \alpha$ in emission), as was reported earlier by Schwope (2001).

\subsection{HS0922+1333}

In our small survey we also included HS0922+1333 (Reimers \& Hagen 2000), because so far it is the only system with similar cyclotron spectra and extremely low mass transfer rate with a period which is above the 2-3 hour period range. Our main goal, as in the case of HS1023+3900, was to check if it shows high luminosity state episodes. The results so far are negative. However, an analysis of its orbital parameters yielded the orbital period directly from the $\mathrm{H} \alpha$ emission line and the $\mathrm{NaI}$ absorption doublet.

The radial velocity measurements of $\mathrm{H} \alpha$ in emission as well as of $\mathrm{NaI}$ absorption doublet folded with period reported by Reimers \& Hagen (2000) showed an obvious discontinuity. Although our coverage was barely enough to overlap periods and it was not possible to estimate the period precisely, the quality of data was good enough to unambiguously establish that the orbital period exceeds the spin period by at least 30 minutes and is of order of 4.6 hours. The narrow band flux measurements centered on the cyclotron emission lines and folded on either the newly determined orbital, and the previously reported 4.07 hour periods confirm that the latter is a better fit and so this most probably 

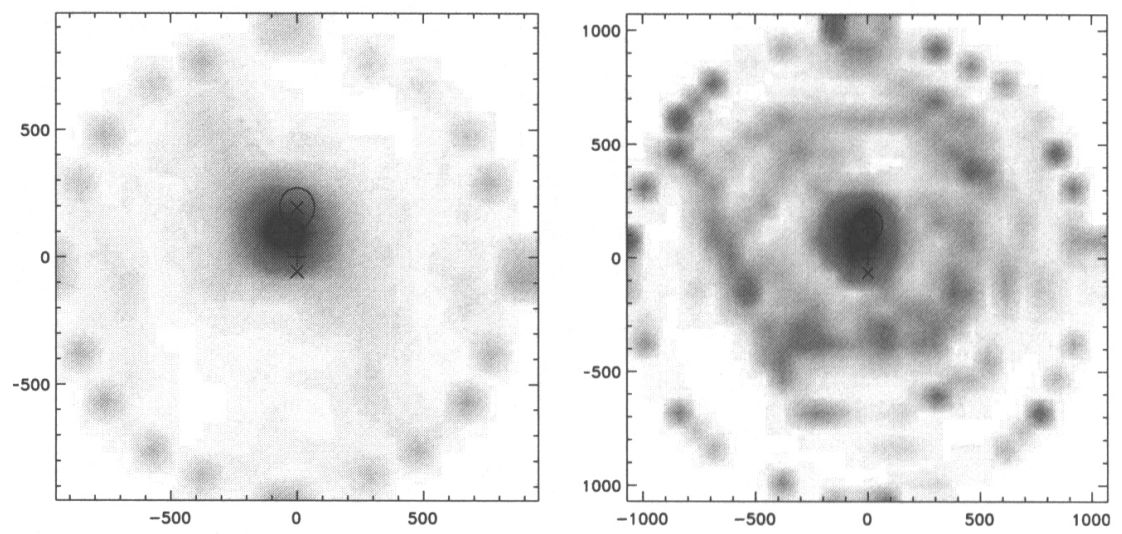

Figure 6. Doppler maps of HS0922+1333. $\mathrm{H} \alpha$ in the left panel, $\mathrm{NaI}$ in the right

represents the spin period of the primary. In Figure 5 (similar to Figure 2) the radial velocity and flux measurements are folded with the proposed orbital and spin periods. The overlapping of phases occurs around $\phi \sim 0.1-0.2$. The lack of synchronization was suspected by the discoverers of the object.

Doppler tomograms of $\mathrm{H} \alpha$ (left) and NaI (right) lines demonstrate the presence of an irradiated secondary star in the system and also some evidence of a mass transfer stream immediately near the $\mathrm{L}_{1}$ point. This is an interesting observation taking into account the meager mass transfer rate of $3 \times 10^{-13} \mathrm{M}_{\odot} /$ year.

\section{Conclusions}

We were able to demonstrate that Polars located in the period gap show all range of spectrophotometric features proper to MCVs:

V381 Vel was observed only in the high state. It shows a significant contribution from the accreting matter flow in emission lines and no secondary irradiation. There is no evidence of significantly low mass transfer rate in this case. It is probably an asynchronous rotator. RXJ1554.2+2721 was frequently observed switching from high to low states. In the high state it shows bluer continuum, strong emission lines both from the irradiated secondary and the stream. In the low state it becomes similar to the LARPs. It is probably synchronous. It bridges the seemingly large differences between LARPS (Schwope 2001) and ordinary Polars. HS1023+3900 has never been observed in high state. The secondary is irradiated. It is probably synchronous.

In the low states the last two objects show extremely low accretion rates $\left(10^{-13} \mathrm{M}_{\odot} /\right.$ year), but so does HS0922+1333 with a period of 4.6 hours and RBS 206 (with a period probably of 90 minutes). Thus, we may speak about existence of a distinct class of Polars with a lower mass transfer rates than originally known. However there is no strong evidence linking low mass transfer rate with the "classical" period gap. The objects we have considered have magnetic field strengths and secondaries whose spectral classes are within the usual range of values. 


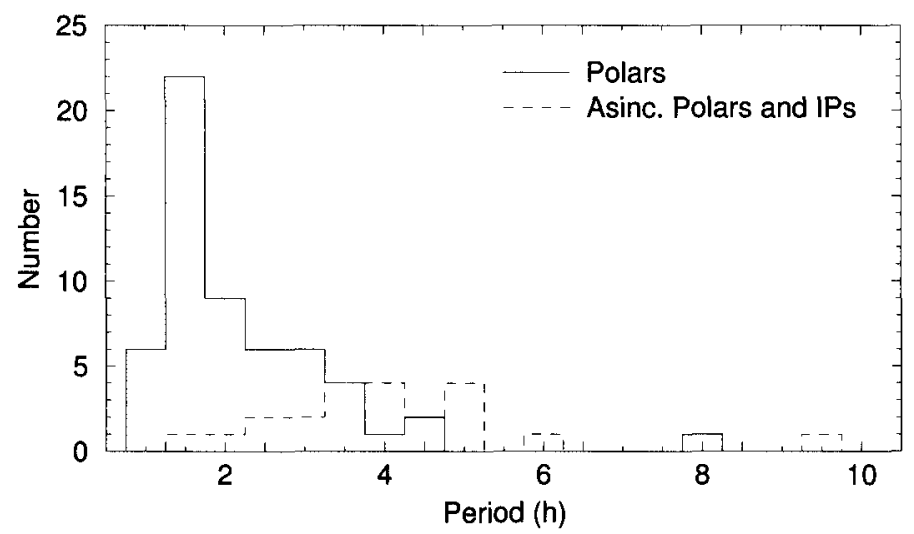

Figure 7. Orbital period distribution of MCVs

Redrawing the orbital distribution graph with the new assignments (HS0922+1333 and V381 Vel as asynchronous polars) creates an impression that the 2-3 hour range is a "melting point" where magnetic systems reach synchronization. This conclusion is in a good agreement with the prediction drawn by Webbink \& Wickramasinghe (2002) from their models (although they expect at least $10^{-10}$ $\mathrm{M}_{\odot} /$ year). LARPs are still understudied objects and they can provide important insights to the nature of MCVs.

Discovery of a couple of highly asynchronous polars arises the question of the definition of Intermediate Polars. Are they simply MCVs with a spin period of the primary sufficiently different from the orbital period or is the strength of magnetic field the most important defining parameter?

Acknowledgments GHT acknowledges partial financial support received from IAU to take part in the IAU Colloquium on MCVs.

\section{References}

Downes, R., Webbink, R.F. \& Shara, M. 1997, PASP, 109, 345

Greiner, J., \& Schwarz, R. 1998, A\&A, 340,129

Reimers, D., Hagen, H.-J., \& Hopp, U. 1997, A\&A, 343,157

Reimers, D., \& Hagen, H.-J. 2000, A\&A, 358, L45

Schwope, A.D., Brunner, H., Hambaryan, V., \& Schwarz, R. 2002, in The Physics of CVs and Related Objects, ASP Conf. Ser., 261, 102.

Thorstensen, J.R., \& Fenton, W.H. 2002, PASP, 114, 74

Tovmassian, G.H., Greiner, J., Zharikov, S.V., Echevarria, J., \& Kniazev, A. 2001, A\&A, 380, 504

Tovmassian G.H., Mennickent, R., Greiner, J., \& Zarikov, S.V. 2003a, in prep.

Tovmassian, G.H., Zarikov, S.V., \& Mercado, A. 2003b, in prep.

Vennes, S., Ferrario, L., \& Wickramasinghe, D. 1999, Annapolis Workshop on Magnetic CVs, ASP Conf. Ser., 157, 143

Webbink, R.F. \& Wickramasinghe, D.T. 2002, MNRAS, 335, 1 